\title{
Time evolution of a quantum many-body system: transition from integrability to ergodicity in thermodynamic limit
}

\author{
Tomaž Prosen \\ Physics Department, Faculty of Mathematics and Physics, University of Ljubljana, Jadranska 19, 1111 Ljubljana, Slovenia
}

(September 7, 2018)

Numerical evidence is given for non-ergodic (non-mixing) behavior, exhibiting ideal transport, of a simple nonintegrable many-body quantum system in the thermodynamic limit, namely kicked $t-V$ model of spinless fermions on a ring. However, for sufficiently large kick parameters $t$ and $V$ we recover quantum ergodicity, and normal transport, which can be described by random matrix theory.

PACS numbers: 05.30.Fk, 05.45.+b, 72.10.Bg

A simple question is addressed here: "Do mixed quantum many-body systems, which are neither integrable nor ergodic, exist in the thermodynamic limit?" While it is clear that integrable systems are rather exceptional it is an important open question whether a finite generic perturbation of an integrable system becomes ergodic or not in the thermodynamic limit (TL), size $\rightarrow \infty$ and fixed density. It is known that local statistical properties of quantum systems with few degrees of freedom whose classical limit is completely chaotic - ergodic are universally described by random matrix theory, while in the other extreme case of integrable systems Poissonian statistics may typically be applied [1,2]. This statement has recently been verified numerically also for integrable and strongly non-integrable many-body systems of interacting fermions [3] which do not have classical limit.

Having lost the reference to classical dynamics we resort to the definition of quantum ergodicity (also termed quantum mixing) [4] as the decay of time-correlations $\langle A(\tau) B(0)\rangle-\langle A\rangle\langle B\rangle$ of any pair of quantum observables $A$ and $B$ in TL, taking the time-limit $\tau \rightarrow \infty$ in the end. In [4] many-body system of interacting bosons has been studied and it has been shown that quantum ergodicity corresponds to strongly chaotic (classically ergodic) dynamics of associated non-linear mean-field equations. As a consequence of linear response theory, quantum ergodicity also implies normal transport and finite transport coefficients (such as dc electrical conductivity). On the other hand, integrable systems, which are solvable by Bethe ansatz or quantum inverse scattering, are characterized by (infinitely many) conservation laws and are thus non-ergodic. It has been pointed out recently [5] that integrability implies nonvanishing stiffness, i.e. ideal conductance with infinite transport coefficients (or ideal insulating state). As we argue below, any deviation from quantum ergodicity generically implies nonvanishing long-time current auto-correlation and therefore infinite transport coefficient. Since generic non-integrable systems of finite size (number of degrees of freedom) are non-ergodic (obeying mixed statistics smoothly interpolating from Poissonian to random matrix results) it is thus an important question if and when such nonergodicity can survive TL.

In this Letter we introduce a family of simple manybody systems smoothly interpolating between integrable and ergodic regime, namely kicked $t$ - $V$ model (KtV) of spinless fermions with periodically switched nearest neighbor-interaction on a 1-dim lattice of size $L$ and periodic b.c. $L \equiv 0$, with time-dependent hamiltonian

$$
H(\tau)=\sum_{j=0}^{L-1}\left[-\frac{1}{2} t\left(c_{j}^{\dagger} c_{j+1}+h . c .\right)+\delta_{p}(\tau) V n_{j} n_{j+1}\right],
$$

and give numerical evidence for the existence of mixed regime (reader should not confuse it with mixing) in TL by direct simulation of the time evolution. $c_{j}^{\dagger}, c_{j}, n_{j}$ are fermionic creation, annihilation and number operators, respectively, and $\delta_{p}(\tau)=\sum_{m=-\infty}^{\infty} \delta(\tau-m)$. Deviations from quantum ergodicity (or mixing) are characterized by several different quantities as described below.

$\mathrm{KtV}$ model (1) is a many-body analogue of popular 1dim non-integrable kicked systems [2] such as e.g. kicked rotor: its evoulution (Floquet) operator over one period $U=\hat{T} \exp \left(-i \int_{0^{+}}^{1^{+}} d \tau H(\tau)\right)(\hbar=1)$, factorizes into the product of kinetic and potential part

$$
U=\exp \left(-i V \sum_{j=0}^{L-1} n_{j} n_{j+1}\right) \exp \left(i t \sum_{k=0}^{L-1} \cos (s k+\phi) \tilde{n}_{k}\right)
$$

where $s=2 \pi / L$. Flux parameter $\phi$ is used in order to introduce a current operator $J=\left.(i / t) U^{\dagger} \partial_{\phi} U\right|_{\phi=0}=$ $\sum_{k=0}^{L-1} \sin (s k) \tilde{n}_{k}$, elsewhere we put $\phi:=0$. Tilde denotes the operators which refer to momentum variable $k$, $\tilde{c}_{k}=L^{-1 / 2} \sum_{j=0}^{L-1} \exp (i s j k) c_{j}, \tilde{n}_{k}=\tilde{c}_{k}^{\dagger} \tilde{c}_{k}$. KtV model is integrable if either $t=0$, or $V=0 \quad(\bmod 2 \pi)$, or $t V \rightarrow 0$ and $t / V$ finite (continuous time $\mathrm{t}-\mathrm{V}$ model, see e.g. [6]), while for $t \sim V \sim 1$ it is expected to be non-integrable, either quantum ergodic or mixed.

We expect that unitary many-body quantum maps, such as (2), also mimick the dynamics of generic autonomous quantum many-body systems on the energy shell in a similar way as 1-dim quantum maps describe (quantum) Poincaré sections of 2-dim quantum (chaotic) systems (see e.g. [8]). 
The total number of particles $N=\sum_{j} n_{j}$ is conserved, so the map $U$ acts over Hilbert (Fock) space $\mathcal{H}$ of dimension $\mathcal{N}=\left(\begin{array}{l}L \\ N\end{array}\right)$. The dynamics of a given initial many body state $|\psi(0)\rangle$, which is an iteration of the map $|\psi(m)\rangle=U|\psi(m-1)\rangle=U^{m}|\psi(0)\rangle$, can be performed most efficiently by observing that the kinetic part $U_{T}$ is diagonal in the momentum basis $|\vec{k}\rangle=\tilde{c}_{k_{1}}^{\dagger} \cdots \tilde{c}_{k_{N}}^{\dagger}|0\rangle, k_{1}<$ $\ldots<k_{N}$ while the potential part $U_{V}$ is diagonal in the position basis $|\vec{j}\rangle=c_{j_{1}}^{\dagger} \cdots c_{j_{N}}^{\dagger}|0\rangle, j_{1}<\ldots<j_{N}$. The transformation between the two, $F_{\vec{j} \vec{k}}=\langle\vec{j} \mid \vec{k}\rangle$, is an antisymmetrized N-dim discrete Fourier transformation (DFT) on L-sites which has been efficiently coded in $\sim \mathcal{N} \log _{2} \mathcal{N}$ floating point operations (FPO) by factorizing $L$-site DFT to the product of $\mathcal{O}\left(L \log _{2} L\right) 2$ site transformations parametrized with $2 \times 2$ sub-matrices $(\alpha, \beta ; \gamma, \delta)_{j j^{\prime}}$, which are successively applied to creation operators, $\left(c_{j}^{\dagger}, c_{j^{\prime}}^{\dagger}\right) \leftarrow\left(\alpha c_{j}^{\dagger}+\beta c_{j^{\prime}}^{\dagger}, \gamma c_{j}^{\dagger}+\delta c_{j^{\prime}}^{\dagger}\right)$, in all slater determinants $\Pi_{n} c_{j_{n}}^{\dagger}|0\rangle$ which contain a particle at sites $j$ or $j^{\prime}$. Our algorithm (fermionic FFT) requires almost no extra storage apart from a vector of $\mathcal{N}$ c-numbers and works for lattices of sizes $L=2^{p}, 10,12,15,20,24,30,40$. Therefore, the map (2) is iterated on a vector $\psi_{\vec{k}}(m)=$ $\langle\vec{k} \mid \psi(m)\rangle$, using the matrix composition $U=F^{*} U_{V} F U_{T}$ in roughly $2 \mathcal{N} \log _{2} \mathcal{N}$ FPO per time step which is by far superior to complete diagonalization techniques $\left(\mathcal{O}\left(\mathcal{N}^{3}\right)\right.$ FPO), even for long time scales $m=\mathcal{O}(\mathcal{N})$ when quantum dynamics becomes quasiperiodic due to discreteness of the spectrum of $U$.

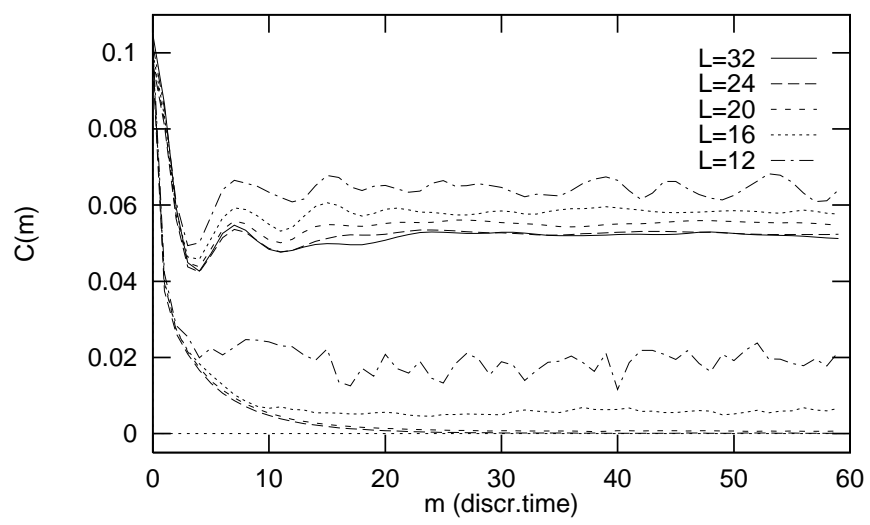

FIG. 1. Current autocorrelation function $C_{J}(m)$ against discrete time $m$ for quantum ergodic $(t=V=4$, lower set of curves for various sizes $L)$ and mixed regime $(t=V=1$, upper set of curves) with density $\rho=\frac{1}{4}$. Averaging over entire Fock space is performed, $\mathcal{N}^{\prime}=\mathcal{N}$, for $L \leq 20$, whereas random samples of $\mathcal{N}^{\prime}=12000$, and $\mathcal{N}^{\prime}=160$ initial states have been used for $L=24$, and $L=32$, respectively.

First we consider decay of current autocorrelation function $C_{J}(m)=(1 / L)\langle J(m) J(0)\rangle$ where $J(m)=$ $U^{\dagger m} J U^{m}$, and $\langle\rangle=.(1 / \mathcal{N}) \operatorname{Tr}($.$) is a 'microcanonical av-$ erage'. Note that $J$ is diagonal in the momentum basis $J|\vec{k}\rangle=J_{\vec{k}}|\vec{k}\rangle$, and $\langle J\rangle=0$. So $C_{J}(m)$ can be evaluated by means of time-evolution of momentum initial states $|\psi(0)\rangle=\left|\vec{k}^{\prime}\right\rangle$

$$
C_{J}(m)=\frac{1}{L \mathcal{N}^{\prime}} \sum_{\vec{k}^{\prime}}^{\prime} J_{\vec{k}^{\prime}} \sum_{\vec{k}} J_{\vec{k}} p_{\vec{k} \vec{k}^{\prime}}(m)
$$

where $p_{\vec{k} \vec{k}^{\prime}}(m)=|\langle\vec{k} \mid \psi(m)\rangle|^{2}=\left|\left\langle\vec{k}\left|U^{m}\right| \vec{k}^{\prime}\right\rangle\right|^{2}$. For large sizes $L$, a smaller but uniformly random sample of $\mathcal{N}^{\prime}$ initial states $\left|\vec{k}^{\prime}\right\rangle, 1 \ll \mathcal{N}^{\prime} \ll \mathcal{N}$, is used in order to save computer time. Direct computation of $C_{J}(m)$ for $m \leq M$ can be performed in $\sim\left(2 M \mathcal{N N}^{\prime} / L\right) \log _{2} \mathcal{N}$ FPO, since due to translational symmetry one can simultaneously simulate the dynamics of $L$ different states with different values of the conserved total momentum $K=\sum_{n} k_{n}^{\prime}$ $(\bmod L)$. Using the eigenphases $\eta_{n}$ and eigenstates $|n\rangle$ of evolution operator $U, U|n\rangle=e^{-i \eta_{n}}|n\rangle, n=1 \ldots \mathcal{N}$, one can write dissipative dc conductivity of such a kicked system $\sigma:=\sum_{n=1}^{\mathcal{N}}\left(\partial_{\phi} \eta_{n}\right)^{2} \approx C_{J}(0)+2 \sum_{m=1}^{\mathcal{N} / 2} C_{J}(m)$. Note that $\partial_{\phi} \eta_{n}=\langle n|J| n\rangle$.

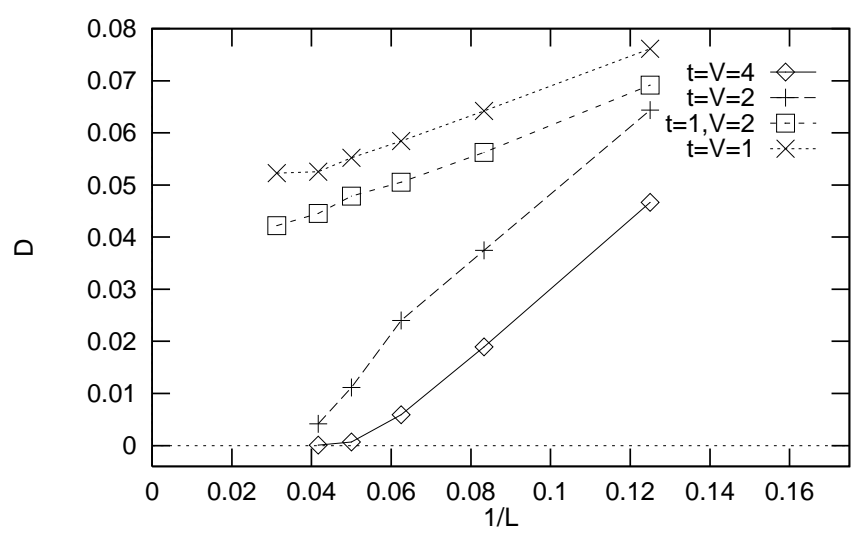

FIG. 2. Stiffness $D_{J}$ vs. $1 / L$ at constant density $\rho=\frac{1}{4}$ and for different values of control parameters in ergodic, $t=V=4$ and $t=V=2$, and mixed, $t=1, V=2$ and $t=V=1$, regime. Other parameters are the same as in fig.1.

In fig.1 we present numerical computation of correlation function $C_{J}(m)$ for parameters $t=V=1$ and $t=V=4$, for various sizes $L$, but at fixed density $\rho=N / L=\frac{1}{4}$. Quite generally, $C_{J}(m)$ exhibits fast relaxation on a time scale $M^{*}$ which is typically small, $M^{*} \sim 10$, and roughly independent of $L$, and afterwards it fluctuates around averaged limiting value, the stiffness

$$
D_{J}=\lim _{M \rightarrow \infty} \frac{1}{M} \sum_{m=1}^{M} C_{J}(m)
$$

where the strength of fluctuations decreases with incresing size $L$. Note again that TL $L \rightarrow \infty$ should be taken prior to the time-limit, $\lim _{M \rightarrow \infty}(1 / M) \sum_{m=1}^{M}($.$) ,$ which is for systems of finite size $L$ here and below estimated numerically as $\left(1 / M^{\prime}\right) \sum_{m=M^{\prime}+1}^{2 M^{\prime}}($.$) with suffi-$ ciently large but fixed averaging time $M^{\prime}>M^{*}$; we 
take $M^{\prime}=30$. If the system is quantum ergodic (case $t=V=4$ of fig.1), $D_{J}$ goes to zero and $\sigma$ remains finite as $L \rightarrow \infty(\mathcal{N} \rightarrow \infty)$ and $\rho=N / L$ fixed, whereas in the other case $(t=V=1$ of fig. 1$) D_{J}$ remains well above zero as we approach TL whereas conductivity $\sigma$ diverges [7]. In fig.2 we have analyzed the scaling of $D_{J}$ with $1 / L$. For large values of parameters, say $t=V=4, D_{J}$ is practically zero already for $L \approx 20$, while for smaller (but not small) control parmeters $(t, V), D_{J} \approx D_{J}^{\infty}+\beta / L$ where $D_{J}^{\infty}>0$. In close-to-critical case $t=V=2$, we find larger correlation time $M^{*} \sim 10^{2}$, and hence use longer averaging time $M^{\prime}=200$. In fig.3 we illustrate an ideal transport for $t \sim V \sim 1$ by plotting a persistent current $J_{\vec{k}^{\prime}}^{p}=\lim _{M \rightarrow \infty}(1 / M) \sum_{m=1}^{M}\left\langle\vec{k}^{\prime}|J(m)| \vec{k}^{\prime}\right\rangle$ vs. the initial current $J_{\vec{k}^{\prime}}$. The normal transport in the ergodic regime $t=V=4$ is characterized by $J_{\vec{k}^{\prime}}^{p}=0$, while for $t \sim V \sim 1$ we find ideal transport with the persistent current being proportional to the initial current, $J_{\vec{k}^{\prime}}^{p}=\alpha J_{\vec{k}^{\prime}}$. Proportionality constant $\alpha$ can be computed from (4) $D_{J}=(1 / L)\left\langle J_{\vec{k}^{\prime}} J_{\vec{k}^{\prime}}^{p}\right\rangle=(\alpha / L)\left\langle J^{2}\right\rangle$, so $\alpha=2 D_{J} /[\rho(1-\rho)]$, where $\left\langle J^{2}\right\rangle$ is given below (5).

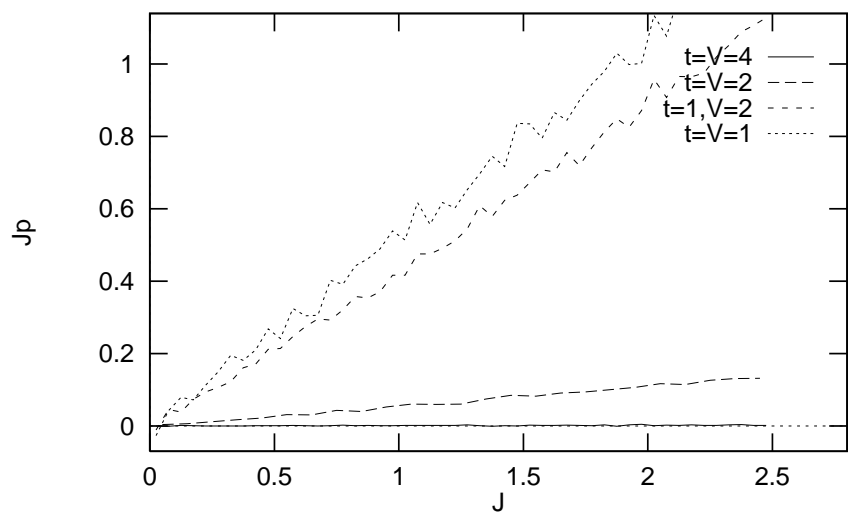

FIG. 3. Persistent current $J_{\vec{k}}^{p}$ against initial current $J_{\vec{k}}$ (averaged over bins of size $\Delta J=0.005$ ) in the ergodic $t=V=4$, (nearly) ergodic $t=V=2$, and mixed regime, $t=V=1$ and $t=1, V=2$. In all cases, $L=24$ and $\rho=\frac{1}{4}$.

Due to translational symmetry the total momentum $K=$ $\sum_{k} k \tilde{n}_{k} \quad(\bmod L)$ is the only conserved quantity (appart from $N$ and parity), so the evolution of initial momentum state $\left|\vec{k}^{\prime}\right\rangle$ takes place in $\mathcal{N}_{K} \approx \mathcal{N} / L \operatorname{dim}$. subspace $\mathcal{H}_{K}$, spanned by $|\vec{k}\rangle$ with $K=|\vec{k}|:=\sum_{n} k_{n}$. Starting with a momentum state $\left|\vec{k}^{\prime}\right\rangle$, the number of 'excited' states $|\vec{k}\rangle$ after time $m$ is characterized by information entropy [9] (see also [8]) as $\exp \left(-\sum_{\vec{k}} p_{\vec{k} \vec{k}^{\prime}}(m) \log p_{\vec{k} \vec{k}^{\prime}}(m)\right)$. Averaging the entropy over uniformly random sample of $\mathcal{N}^{\prime}$ initial states $\left|\vec{k}^{\prime}\right\rangle$ we define relative localization dimension in Fock space as a measure of quantum ergodicity.

$$
R(m)=\frac{L}{\mathcal{N}} \exp \left(-\frac{1}{\mathcal{N}^{\prime}} \sum_{\vec{k}^{\prime}}^{\prime} \sum_{\vec{k}} p_{\vec{k} \vec{k}^{\prime}}(m) \log p_{\vec{k} \vec{k}^{\prime}}(m)\right) .
$$

Again similar behavior is found numerically for $R(m)$ as for $C_{J}(m)$ (the two quantities can be computed simultaneously at no extra cost), namely it typically saturates within the same (short) correlation time $M^{*}$ to a roughly constant value $\bar{R}=\lim _{M \rightarrow \infty}(1 / M) \sum_{m=1}^{M} R(m)$

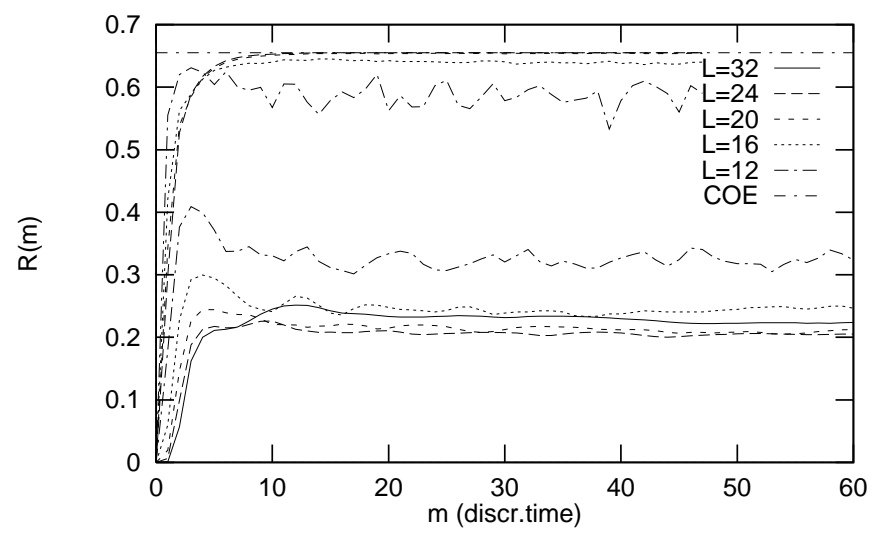

FIG. 4. Relative localization dimension in Fock space, $R(m)$ for data of fig. 1 .

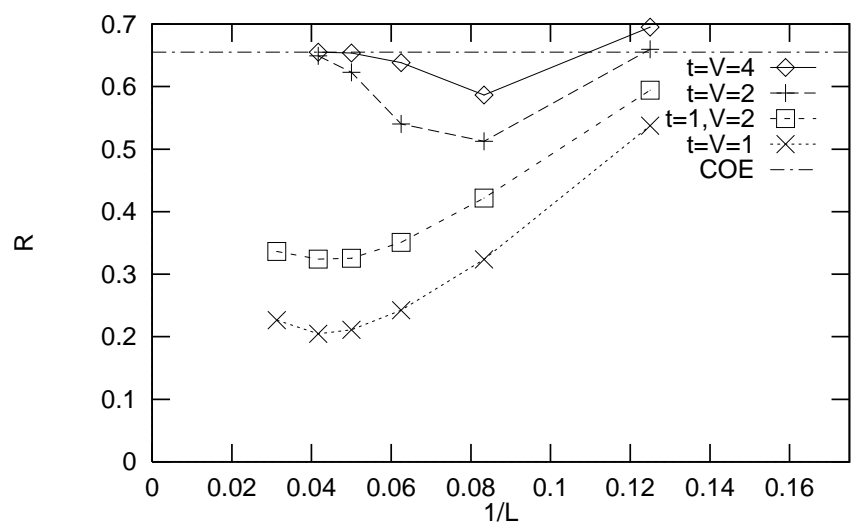

FIG. 5. Limiting rel.loc.dim. $\bar{R}$ vs. $1 / L$ for data of fig.2.

If there are no additional, even approximate conservation laws the blocks $\left.U^{m}\right|_{\mathcal{H}_{K}}$ may be modeled by circular orthogonal ensemble (COE) of random matrices for sufficiently large $m$ giving the maximal asymptotic $($ as $\mathcal{N} \rightarrow \infty)$ value of relative localization dimension, $\bar{R}_{C O E} \approx 0.655$. This case corresponds to quantum ergodicity since $p_{\vec{k} \vec{k}^{\prime}}(m)$, for $m>M^{*}$, become pseudo random and independent of $\vec{k}$ and $\vec{k}^{\prime}$, hence the correlation function (3) factorizes and yields $C(m)=\langle J\rangle^{2}=0$. Indeed, as we show in fig.4, such behavior is obtained only for sufficiently large parameters, say $t=V=4$, while for smaller values of parameters $t, V, R(m)$ saturates to a smaller value indicating that there may exist approximate conservation laws causing nontrivial localization inside the Fock space. Scaling with $1 / L$ suggests that even TL of $\bar{R}$ is smaller than $\bar{R}_{C O E}$ for $t \sim V \sim 1$ (fig.5). 


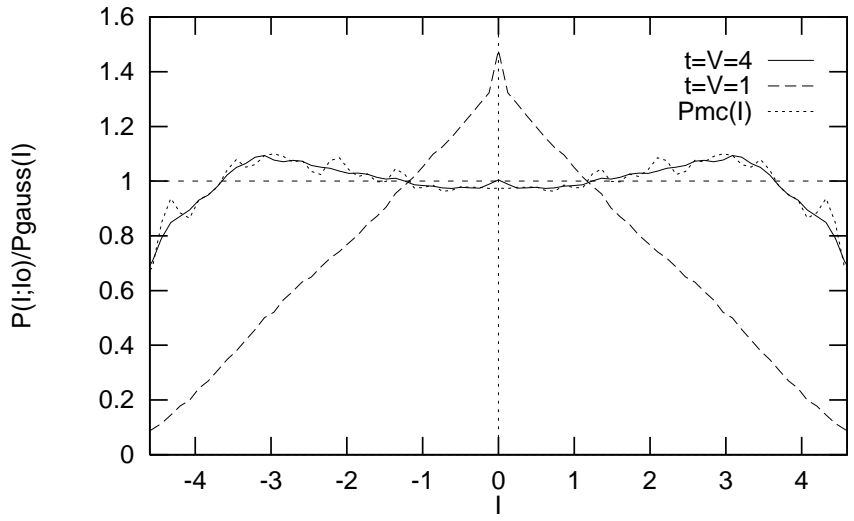

FIG. 6. Steady-state current distribution divided by a gaussian $P\left(I, I_{0}\right) / P_{\text {gauss }}(I)$ averaged over 279 initial orbits with $\left|I_{0}\right|<0.08$ in the ergodic, $t=V=4$, and mixed, $t=V=1$, regime, and the finite-size microcanonical current distribution $P_{m c}(I)$. $L=24, \rho=\frac{1}{4}$.

Finally, we discuss current fluctuations, or more generaly, current distribution $P_{\psi}(I)=\langle\psi|\delta(I-J)| \psi\rangle$ giving a probability density of having a current $I$ in a state $|\psi\rangle$. We let the state $\psi$ with a 'good' known initial current $I_{0}$ to evolve for a long time from which we compute a stady-state current distribution (SSCD)

$$
P\left(I ; I_{0}\right)=\lim _{M \rightarrow \infty} \frac{1}{M} \sum_{m=1}^{M}\left\langle\delta\left(I_{0}-J(0)\right) \delta(I-J(m))\right\rangle .
$$

Of course, delta functions should have a finite small width providing averaging over several states $|\vec{k}\rangle$ with $J_{\vec{k}} \approx I_{0}$. In the quantum ergodic regime all states eventually become populated, so $\operatorname{SSCD} P\left(I ; I_{0}\right)$ should be independent of initial current $I_{0}$ and equal to the $m i$ crocanonical current distribution $P_{m c}(I)=\langle\delta(I-J)\rangle$. It has been shown by elementary calculation that in TL the latter becomes a gaussian, $P_{m c}(I) \rightarrow P_{\text {gauss }}(I)=$ $\left(1 / \sqrt{2 \pi\left\langle J^{2}\right\rangle}\right) \exp \left(-\frac{1}{2} I^{2} /\left\langle J^{2}\right\rangle\right)$, while at any finite size $L$ the first few moments are:

$$
\begin{aligned}
\left\langle J^{2}\right\rangle & =\frac{N(L-N)}{2(L-1)} \approx \frac{1}{2} \rho(1-\rho) L \\
\frac{\left\langle J^{4}\right\rangle}{\left\langle J^{2}\right\rangle^{2}} & =\frac{3(L-1)(2 N(L-N)-L)}{2 N(L-2)(L-N)} \\
& =3+\frac{3(2 \rho(1-\rho)-1)}{2 \rho(1-\rho)} \frac{1}{L}+\mathcal{O}\left(L^{-2}\right) .
\end{aligned}
$$

Numerical results for $L=24$ (see fig.6) indicate that in the ergodic regime, $t=V=4, \mathrm{SSCD}$ is already in good agreement with microcanonical distribution $P_{m c}(I)$, while in non-ergodic (mixed) regime, $t=$ $V=1$, SSCD is localized on a smaller range indicating that the current fluctuation is smaller than $\left\langle J^{2}\right\rangle$. Note that the mean $\bar{I}=\int d I I P\left(I ; I_{0}\right)$ is just a persistent current, so $\bar{I}=\alpha I_{0}$ (see fig.3).
In this Letter we have presented numerical evidence, based on efficiently coded time evolution of a kicked fermionic system, in support of hypothesis that mixed (neither integrable nor ergodic) behavior of a quantum many-body system may survive TL provided that control parameters are not too far away from integrable points. It has been shown that in this regime ideal transport is possible. However, if the control parameters are sufficiently large we recover quantum ergodycity compatible with random matrix theory and normal transport properties. It is interesting to note that at the transition point between the two regimes, where order parameter - stiffness $\left.D_{J}\right|_{L=\infty}$ (inferred from $1 / L$ scaling) touches zero, the correlation time scale $M^{*}$ drastically increases what is reminiscent of a kind of dynamical phase transition. Although only data for quarter-filled lattice $\left(\rho=\frac{1}{4}\right)$ are presented here, we should stress that essentially the same conclusions follow from our data for other densities, $\rho=\frac{1}{3}, \frac{3}{8}, \frac{2}{5}, \frac{1}{2}$, with a general rule, that the border of quantum ergodic regime moves to slightly smaller values of control parameters $t, V$ as the density $\rho$ approaches $\frac{1}{2}$. It should be noted that statistics of eigenphases of evolution operator $U$ has been computed as well and it has been found that in the ergodic regime level statistics is indeed that of $\mathrm{COE}$ while in the mixed regime it smoothly interpolates between Poisson and COE.

Discussions with Prof. P. Prelovšek, and the financial support by Ministry of Science and Technology of the Republic of Slovenia are gratefully acknowledged.

[1] O.Bohigas, M.-J.Giannoni, and C.Schmit, Phys.Rev.Lett. 52, 1 (1984); A.V.Andreev, O.Agam,B.D.Simons, and B.L.Altshuler, Phys.Rev.Lett. 76, 3947 (1996).

[2] F.Haake, Quantum Signatures of Chaos (Springer-Verlag, Berlin-Heidelberg 1991).

[3] G.Montambaux, D.Poilblanc, J.Bellisard, and C.Sire, Phys.Rev.Lett.70, 497 (1993); T.C.Hsu and J.C.Angles d'Auriac, Phys.Rev.B 47,14291 (1993); D.Poilblanc, T.Ziman, J.Bellisard, F.Mila, and G.Montanbaux, Europhys.Lett.22,537 (1993).

[4] G.Jona-Lasinio and C.Presilla, Phys.Rev.Lett.77, 4322 (1996).

[5] X.Zotos and P.Prelovšek, Phys.Rev.B 53, 983 (1996); H.Castella, X.Zotos, and P.Prelovšek, Phys.Rev.Lett. 74, 972 (1995).

[6] V.J.Emery, in Highly conducting one-dimensional solids, ed. J.T.Devreese, R.P.Evrard and V.E. van Doren (Plenum Publishing Corporation, 1979).

[7] Note that the case $t=V=1$ is far beyond perturbative treatement of any integrable case. For example, statistics of eigenphases $\eta_{n}$ exhibit substantial level repulsion.

[8] T.Prosen, Physica D91, 244 (1996)

[9] F. Izrailev, J.Phys.A 22, 865 (1989). 